\title{
NRAS NM_002524.4:c.180_181delACinsTA
}

National Cancer Institute

\section{Source}

National Cancer Institute. NRAS NM 002524.4:C.180 181delACinsTA. NCI Thesaurus.

Code $C 98447$.

A complex substitution where the nucleotide sequence at positions 180 through 181 of the coding sequence of the NRAS gene has changed from adenine-cytosine to thymineadenine. 